\title{
Efficacy of dolutegravir-based antiretroviral regimens in perinatally HIV-1-infected youth with long-term virological failure.
}

\section{T. Puthanakit ${ }^{1,2}$, S. Anugulruengkitt ${ }^{1,2}$, W. Jantarabenjakull,2, W.N. Songtaweesin ${ }^{2}$, J. Sophonphan ${ }^{3}$, C. Saisangjan ${ }^{4}$}

${ }^{1}$ Department of Pediatrics, Bangkok, Thailand. ${ }^{2}$ Center of Excellence in Pediatric Infectious Diseases and Vaccines, Chulalongkorn University, Bangkok, Thailand. ${ }^{3}$ HIV-NAT, ${ }^{4}$ SEARCH, Thai Red Cross AIDS Research Center, Bangkok, Thailand.

\section{Background}

- Dolutegravir (DTG) is a potent antiretroviral drug in an integrase transfer

inhibitor class with high potency and convenient as a once-daily regimen. ${ }^{1}$

- Generic dolutegravir is available since 2017, but access in Asia is limited. ${ }^{2}$

\section{Objective}

- To assess the efficacy of a dolutegravir-based regimen in HIVinfected youth with persistent virological failure with PI regimens

\section{Methods}

Design: A prospective observational cohort

Settings: King Chulalongkorn Memorial Hospital and HIV-NAT, Bangkok. Inclusion criteria

- Perinatally HIV-infected youth aged 15-24 years

- Failing second-line boosted protease inhibitors (bPIs) for $>2$ years

\section{Study procedure}

\section{Antiretroviral regimens}

A. No major PI resistance mutations: Dolutegravir/Tenofovir/Emtricitabine

B. With major PI RAMS: Dolutegravir/Darunavir/r/Tenofovir/Emtricitabine

Endpoints

- Proportion of patients achieving plasma HIV $<200$ and $<1000$ copies $/ \mathrm{mL}$

- CD4 T lymphocyte cell count change over time

\section{Results}

- From January to October 2017, 15 perinatally HIV-infected youth were enrolled (Table1).

- The previous bPI regimens were Atazanavir/r(53\%), Lopinavir/r(27\%), Darunavir/r(20\%).

- 11 youth received regimen A (DTG-based) and 4 youth regimen B (DTG+DRV/r-based)

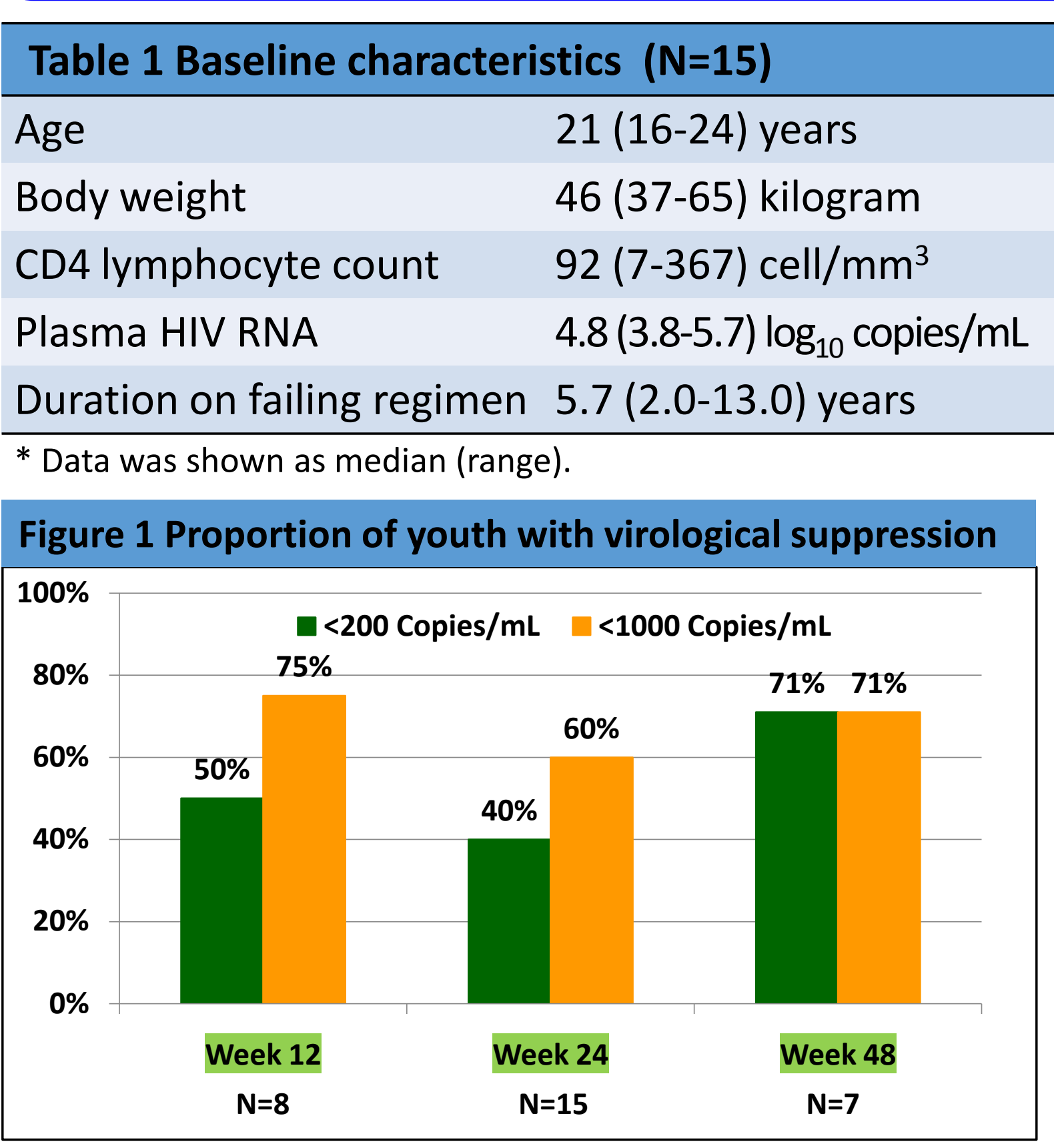

Contact person: Thanyawee Puthanakit, MD E-mail: thanyawee.p@chula.ac.th

\section{Conclusions}

- Dolutegravir-based regimens can be utilized effectively as rescue regimens for youth who are failing a second-line PI-based regimen.

- Access to generic dolutegravir is urgently needed for HIV-infected youth with treatment failure in resource-limited settings.

\section{Acknowledgement}

The generic dolutegravir access program was funded by donation program at the Thai Red Cross AIDS research center.

\section{References}

1. Briand C, Dollfus C, Fay A, et al. Efficacy and tolerance of dolutegravir-based combined ART in perinatally HIV-1infected adolescents: a French multicentre retrospective study. J Antimicrob Chemother 2017;72:837-843. 2. Cohn J, Bekker LG, Bygrave H, et al. Hit me with your best shot: dolutegravir - a space in the next WHO guidelines? AIDS. 2015;29:2067-70. 